\title{
Alfonsina Storni oder die Entfaltung einer feministischen Lyrik
}

Beschäftigen wir uns vor einer Auseinandersetzung mit dem Werk und einzelnen Texten zunächst mit der Biographie der argentinischen Lyrikerin Alfonsina Storni, die in gewisser Weise zu jenen Einwanderern gehört, die dem Argentinien des 20. Jahrhunderts ein völlig neues Aussehen, eine neue Struktur und neue Hoffnungen auf ein besseres Leben gaben. Es sind Jahre intensiver sozialer Kämpfe und Auseinandersetzungen, aber auch wachsender wirtschaftlicher Macht und der Gestaltung jener sozialen, politischen und ökonomischen Strukturen, die das Land in eine dauerhafte wirtschaftliche und gesellschaftliche Krisensituation führen sollten. Doch beschäftigt man sich mit Alfonsina Storni, so rücken nicht die gesellschaftlichen Eliten in den Blickpunkt, welche die Verantwortung für den späteren Niedergang des Landes tragen, sondern anders als bei Juana de Ibarbourou die unterprivilegierten sozialen Schichten.

Alfonsina Storni wurde am 29. Mai 1892 in Sala Capriasca im schweizerischen Tessin geboren und starb am 25. Oktober 1938 durch Selbstmord im argentinischen Mar del Plata. Mit ihren Eltern lebte sie bis 1896 im Tessin, dann kehrte die Familie nach San Juan in Argentinien zurück, wo die Stornis eine kleine Fabrik besaßen. Das vernachlässigte Unternehmen machte im Jahr 1900 bankrott. Alfonsina musste einen der Trunksucht ergebenen Vater erdulden, dessen Namen sie in ihrem Vornamen trug. Bald ging der ehemals wohlhabende Bierbrauer mit seinem Café Suizo Pleite, die Mutter eröffnete eine kleine Privatschule im eigenen Haus. Nach dem Tod des Vaters 1906 begann die junge Alfonsina, in einer Hutfabrik zu arbeiten und näherte sich den damals in Argentinien sehr starken anarchistischen Kreisen an. Als eine kleine Theatertruppe durch ihre Stadt kam, schloss sich Alfonsina den Schauspielern an und tourte ein Jahr lang mit wechselnden Rollen durch Argentinien.

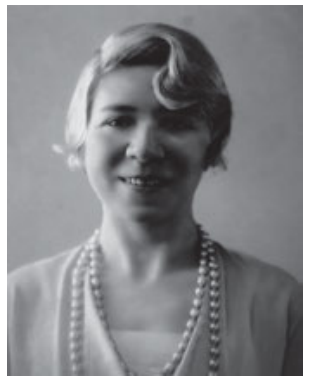

Abb. 89: Alfonsina Storni Martignoni (Sala Capriasca, Bezirk Lugano, Schweiz, 1892 - Mar del Plata, Argentinien, 1938). 
Ein Jahr später jedoch trat sie in die Fußstapfen ihrer Mutter, startete eine Ausbildung als Lehrerin und begann 1911 in Rosario zu unterrichten. Während ihrer Ausbildung arbeitete sie als Sängerin in einem Theater, was für einen Skandal sorgte, der sie erstmals an den Rand eines Selbstmords brachte. Als sie ein uneheliches Kind mit einem verheirateten Politiker erwartete, übersiedelte sie aus der Provinz nach Buenos Aires. Mit verschiedenen Büroarbeiten und anderen Tätigkeiten sorgte sie für ihren Lebensunterhalt und den ihres Sohnes Alejandro. In diesen Jahren erschienen erste Gedichte in lokalen Zeitungen, denen $1916 \mathrm{ihr}$ erster Gedichtband La inquietud del rosal folgte, dessen Kosten sie finanzierte und über lange Jahre abbezahlte.

Ab 1917 verbesserten sich ihre wirtschaftlichen Verhältnisse: Sie wurde Leiterin eines privaten Internats, unter anderem Mitarbeiterin der Periodika Nosotros, Atlántida sowie der einflussreichen Tageszeitung La Nación; ja sie erhielt 1922 den zweiten Premio Nacional für ihren Gedichtband Languidez. Der Ausbruch einer Nervenkrise zwang sie, ihre Stellung im Internat aufzugeben; sie arbeitete zeitweilig als Aufseherin in einer Schule für geistig behinderte Kinder, für die sie mit ihren Geschichten und Liedern da war. Auf einer kleinen Vortragsreise nach Uruguay lernte sie in Montevideo Juana de Ibarbourou und den uruguayischen Modernisten José Enrique Rodó kennen, fühlte sich aber nach ihrer Rückkehr nach Buenos Aires einsam und litt stark unter Depressionen. Trotz des Erfolges von Languidez und einer Reihe guter Besprechungen vermochte sie es kaum, sich finanziell über Wasser zu halten. Immer wieder halfen ihr Freunde aus finanziellen Engpässen und besorgten ihr eine Stelle im Teatro Infantil Labardén, wo sie Dramen für Kinder verfasste.

Mitte der zwanziger Jahre organisierte sie in Mar del Plata die Primera Fiesta de la Poesía, wo sie mit einer Gruppe von Dichterinnen auftrat: Für viele wurde sie zusammen mit Delmira Agustini zum Vorbild schreibender Frauen. Zweimal, 1930 und 1932, reiste sie nach Europa, wobei sie auch einen Abstecher in ihren Schweizer Geburtsort unternahm. Auf diesen Reisen konnte sie insbesondere in Spanien große Vortragserfolge verbuchen. Doch 1935 erkrankte sie an Brustkrebs, eine Chemotherapie musste sie abbrechen. In den Jahren 1937 und 1938 nahmen sich zwei ihrer berühmtesten Freunde, die Schriftsteller Horacio Quiroga und Leopoldo Lugones, das Leben. Noch einmal wurde sie im Januar 1938 nach Montevideo eingeladen, wo sie gemeinsam mit Gabriela Mistral und Juana de Ibarbourou das medienwirksam akklamierte große Dreigestirn der lateinamerikanischen Lyrik bildete. In ihren letzten Gedichten klingt indes bereits das Thema des Selbstmords an. Unheilbar krebskrank, nahm sie sich am Strand von Mar del Plata das Leben.

In ihren Gedichten zeichnete sich mehr und mehr ihr Protest gegen die alles beherrschende patriarchalische Gesellschaftsordnung ab. Ihren ersten 
Gedichtband von 1916 hatten noch modernistische Versatzstücke wie Edelsteine, geschwungene Schwanenhälse und allerlei Orientalismen geziert. Doch weitaus radikaler noch als bei Gabriela Mistral machte sich eine Rebellion gegen jede Art geschlechterspezifischer Konventionen immer schärfer in ihren Gedichten bemerkbar. Alfonsina Storni nimmt ihre eigene postmodernistische Entwicklung: Die tragischen Züge vermischen sich in ihrer Lyrik mit deutlich feministischen Akzenten, gerade auch in ihrer Liebeslyrik.

Sie ist von den drei Dichterinnen sicherlich diejenige, die den männlichen Avantgardisten als feministisch denkende Frau am nächsten stand. ${ }^{1}$ Ihre Gedichte wurden zunehmend pessimistisch mit einem Zug ins Sarkastische, blieben stets aber kämpferisch. Ihr letzter Gedichtband Mascarilla y trébol aus ihrem Todesjahr 1938 besteht aus zweiundfünfzig verschlüsselten Anti-Sonetten, die sich immer weiter von den modernistischen und postmodernistischen Schreibweisen entfernen. In ihrer literarischen Entwicklung wurde Alfonsina Storni gerade durch ihren Kampf gegen die vermeintlich weibliche Sanftmut und ihr ausgeprägtes Rebellentum zur Wegbereiterin für eine lateinamerikanische Literatur von Frauen, die sich gegen jegliche patriarchalische Bevormundung zur Wehr setzen. Während die zu Lebzeiten gefeierten und hochverehrten Gabriela Mistral und Juana de Ibarbourou heutzutage Schwierigkeiten haben, mit ihren Gedichten ein zeitgenössisches Publikum zu begeistern, ist Alfonsina Storni eine hochgeschätzte Inspirationsquelle für eine Vielzahl schreibender Frauen weit über ihr Heimatland hinaus. Es schien mir dennoch adäquat und geboten, allen drei Dichterinnen in unserer Vorlesung den ihnen gebührenden Platz einzuräumen.

Bei Alfonsina Storni haben wir es gewiss mit einer gänzlich anderen Biographie als derjenigen Juana de Ibarbourous zu tun. Auf der einen Seite die Uruguayerin aus alter, oligarchischer Familie, auf der anderen die Argentinierin, die im Tessin geboren wurde, als Kind nach Argentinien einwanderte und erst spät die argentinische Staatsbürgerschaft erhielt. Auf der einen Seite die Frau eines wohlhabenden Offiziers in gesicherten Familienverhältnissen, auf der anderen die - wie wir heute sagen würden - alleinerziehende Mutter, die gesellschaftlich teilweise geächtet wurde und in die Großstadt ging, um in der dortigen Anonymität ihr Leben zu leben und zu verdienen. Auf der einen Seite die finanziell Gesicherte und vom raschen Ruhm Emporgetragene, auf der anderen die sich mehr schlecht als recht durchschlagende Frau, deren Gedichte bei weitem nicht jene Breitenwirkung erzielten, wie sie Juana de Ibarbourou mit ihren Gedichtbänden

1 Vgl. Pleitez Vela, Tania: Capítulo 12: Juntando el sol con gran cordura. Huellas vanguardistas en la poesía de Alfonsina Storni. In: Rodríguez gutiérrez, Milena: Casa en que nunca he sido extraña. Las poetas hispanoamericanas: identidades, femenismos, poéticas (Siglos XIX - XXI), S. 140-151. 
gelang. Doch wollen wir an dieser Stelle unsere Gegenüberstellung beenden, die der existenziellen Situation von Menschen ohnedies nicht wirklich gerecht wird. Denn als selbstbewusste Frauen waren beide zu ihrer Zeit in einem strikt patriarchalischen System marginalisiert.

Über das Frau-Sein, über ihr Frau-Sein, haben beide Lyrikerinnen nicht nur nachgedacht, sondern ihrem Nachdenken auch mehrfach poetischen Ausdruck verliehen. Ohne dieses Gedicht ausführlicher interpretieren $\mathrm{zu}$ wollen, will ich Ihnen doch Juana de Ibarbourous Mujer vorstellen, auf das ich Sie bereits aufmerksam gemacht hatte. Es stammt aus ihrem Gedichtband Raíz salvaje, in welchem die uruguayische Lyrikerin erneut auf jene Wurzelmetaphorik zurückgriff, die wir schon kennengelernt hatten. Es ist eine lyrische Kreation, das ursprünglich den Titel Si yo fuera hombre trug:

\footnotetext{
Wär ich ein Mann, welch Übersättigung an Mond, An Schatten, an Schweigen würd' ich fühlen! Wie würd' ich Nacht für Nacht alleine umherwandeln, Über die ruhigen Felder und dem Meere entlang!

Wär' ich ein Mann, welch ein fremder, verrückter, Hartnäckiger Vagabund ich wohl sein würde!

Ein Freund aller langen Wege, die

Weit weg zu gehen locken, und nimmer zurück!

Wenn mich solch' Gelüste nach Weite anwandeln, Welch tiefe Pein gibt's mir, Frau zu sein! ${ }^{2}$
}

In diesem kurzen Gedicht ist die „condición femenina“ dem Leben und den Lebensmöglichkeiten der Männer klar entgegengesetzt: Eine binäre Oppositionsstruktur tut sich auf, die freilich das Andere - das Männliche - nur im Irrealis zur sprachlichen Realisierung kommen lässt. Zwischen Mann und Frau, zwischen „hombre“ und „mujer“, gibt es keine Vermittlung, keine Graustufen: Allein diese Alternative und keinerlei Übergang eröffnet sich dem weiblichen Ich.

In den beiden ersten Strophen von Mujer entsteht freilich keine verkehrte Welt der Geschlechterrollen, sondern vielmehr eine schlichte Umkehrung, bei welcher das lyrische Subjekt, im Titel genannt, ex negativo zum Manne wird und in der männlich grammatikalisch determinierten Form die Geschlechteridentität und deren Rollenvorgaben an- und wahrnimmt. Bemühen wir die Unterscheidung zwischen der biologischen oder anatomischen Ebene von Sex als Geschlecht und der kulturell definierten Ebene von Gender als Geschlechteridentität, dann wird

2 Ibarbourou. Juana de: Mujer. In (dies.): Obras completas, S. 211. 
deutlich, dass dieses Gedicht hier allein - so scheint mir - auf der Gender-Ebene argumentiert: Nichts Biologisches determiniert die Rollenverständnisse und damit Lebensmöglichkeiten von Frau und Mann.

Die kulturell anders definierte Konstruktion des Mannes wird in diesen Versen virtuell erprobt, letztlich aber in den Bereich unerreichbarer „ansias andariegas“ verbannt, an deren Stelle nur die ,pena tan honda“, die tiefe Pein des Frau-Seins tritt. Gibt es keine Rebellion, noch nicht einmal ein Aufbegehren? Aufschlussreich erscheint mir hierbei, dass bezüglich der Geschlechterrollen gerade die freie Bewegung, der freie Zugang zu einer offenen Räumlichkeit, der Zugang auch zum Alleinsein und sich alleine Bewegen hervorgehoben werden. Nur Mond, Schatten und Schweigen - die wir aus dem zuvor interpretierten Gedicht gut kennen - sind nun genießbar: Es bildet sich fast eine Gegenfolie zur Zweisamkeit im Gedicht Las lenguas de diamante. Doch diese Situation ist schwierig zu ertragen, lastet schwer auf dem weiblichen lyrischen Ich.

Das Freisein gerät hier zum Alleinsein in ungehemmter Bewegung, in Einklang und direktem Zugang mit der und zur Natur. Zugleich wird die größere Toleranz an Abweichungen im Bereich der Geschlechteridentität oder Geschlechterkonstruktion Mann ausgekostet, der „extraño“, „loco“ und „vagabundo“ sein kann, ohne doch aus der Gesellschaft ausgeschlossen zu werden. Die Metaphorik des Vagabundierens, des ziellosen Reisens, ja der Reise ohne Wiederkehr steht dabei im Zentrum eines Gegen-Bildes, das in der Tat der überwiegenden Mehrzahl der Frauen nicht zugänglich war - auch wenn es in den Jahrhunderten zuvor immer wieder auch die Lebenserfahrungen reisender Frauen beziehungsweise von Reiseschriftstellerinnen gab.

Eine Lösung aus dieser ,Conditio feminina“ wird indes nicht angeboten: Keine Erlösung erscheint und bringt alles ins Lot. Nein, es gibt noch nicht einmal ein Aufbegehren, und ist die Pein übers Frau-Sein auch noch so tief. Letztlich fällt das Ich wieder zurück in die weibliche Geschlechterrolle, in die eigene Innerlichkeit, in die Tiefe „honda“) des eigenen Leides und Leidens. Es handelt sich um ein „ser“, nicht etwa um ein „estar“: Das Sein ist angesprochen, nicht ein vorübergehender Zustand. Nur auf dieser Ebene erscheint gleichsam implizit die unveränderbare Substanz, die Essenz des Körperlichen, die Unausweichlichkeit des Sex, der körperlichen geschlechtlichen Determination, der das Subjekt auf der Ebene von Gender nicht zu entrinnen vermag. Aus dieser Situation, so scheint es am Ausgang des Gedichts, führen keine gangbaren Wege, gibt es keine Flucht, keine Evasion, keine Reise weit weit weg. Geschlechtlichkeit ist auf den Ebenen von Sex und Gender - im Sinne des von den zeitgenössischen Feministinnen längst angegriffenen Gemeinplatzes - scheinbar unveränderliches (Frauen-) Schicksal.

Auch Alfonsina Storni musste sich wiederholt dieser Frage der Geschlechtlichkeit stellen, konkret auch bei ihrer ersten Europareise von 1930, auf der sie 
gefragt wurde, ob sie nicht lieber ein Mann hätte sein wollen. Ich zitiere hier ihre Antwort nach Andreolas Storni-Biographie (bei Machín Seite 73):

Ich hätte ein Mann zu sein gewollt, jedoch nicht wegen seiner Freiheit. Denn Freiheit besitzt letztlich die Frau, die sich entschließt, frei zu sein. Nicht darum geht es. Das Erste, was an der Frau verführt, ist ihre Schönheit; beim Mann verführt vor allem anderen das Wort. Wie schön ist es doch, durch das Wort zu verführen! Ich hätte aber wegen etwas Größerem Mann sein gewollt: um träumen, sterben und hassen zu können, nicht aber, um verwirklichen, leben, lieben zu können, was mir als feige und weniger interessant erscheint. ${ }^{3}$

Dies ist fürwahr eine erstaunliche Antwort. Sie blendet zunächst die Frage nach dem Sex - mithin nach dem Verführungsvermögen auf der Ebene körperlicher Schönheit - aus, um die Frage ganz eindeutig in Richtung von Gender als sozialer und kultureller Konstruktion zu wenden. Erstaunlich ist dabei vor allem Alfonsina Stornis Wille und Voluntarismus, könne die Frau doch frei sein, wenn sie es nur wolle. Entscheidendes Mittel hierfür ist das Wort und damit eben jenes Schlüsselwort, das wir auch bei Juana de Ibarbourou entdeckt hatten. Sollten wir nun, mit einiger Entfernung, ihr Auftaktgedicht Las lenguas de diamante geschlechterspezifisch interpretieren und genau als Behauptung jener Kommunikationssituation deuten, in welcher es gerade nicht der Mann ist, der das Wort ergreift und die Worte an die Frau richtet? Ist hier das Schweigen des Mannes vielleicht jener Raum, der das Wort der Frau überhaupt erst ermöglicht, ihre Sprache zur Realisierung kommen lässt oder gar treibt? Die Äußerung Alfonsina Stornis wirft also - just in jenem Jahr, in dem die Ibarbourou zur „Juana de América“ gekrönt wurde - ein völlig anderes Licht auf die Problematik des Wortes, eine Problematik, deren göttliche Verankerung wir freilich nicht vergessen dürfen. Auf die Ebene der Geschlechterpolitik heruntergebrochen, bedeutet all dies die Wichtigkeit jener Wendung, die wir auch im Deutschen dafür haben: „Das Wort ergreifen“, „tomar la palabra“, das ist immer ein Stück Freiheit, die gestaltet werden kann.

Wir sollten zugleich verstehen, dass die Freiheit, die sich Alfonsina Storni in einer durch und durch patriarchalischen Gesellschaft herausnahm, eben jene Freiheit war, welche die Gesellschaft in der Geschlechterkonstruktion dem Mann überantwortete, nämlich durch das Wort zu verführen und zu bestimmen. Gleichzeitig musste sie die männliche Geschlechterrolle annehmen, um dieser eben das zu entnehmen, was herkömmlicherweise in ihr angelegt ist. Denn Alfonsina wollte, wie sie es formulierte, nicht vordringlich realisieren, leben und lieben,

3 Storni, Alfonsina, zitiert nach Andreola, Carlos Alberto: Alfonsina Storni: vida, talento, soledad: primera biografía integral y documentada. Buenos Aires: Ed. Puls Ultra,1976, S. 164. 
sondern träumen, sterben und hassen können, wie sie es ihr gefiel. Wir könnten hier fast von einer gezielten Geschlechterverwirrung sprechen, vielleicht gar von einem „Gender Trouble“ im Sinne Judith Butlers. ${ }^{4}$ Denn das Träumen wird wohl traditionell eher den Frauen, das Sterben (etwa den Heldentod) mehr den Männern überantwortet. Alfonsina Storni wollte aber auch über ihren Tod volle Gewalt besitzen. Doch das Hassen? Dies lässt sich schlechterdings nicht einfach geschlechtlich zuordnen im Sinne der Konstruktion einer Geschlechteridentität. Wir könnten an dieser Stelle fast von einer Dekonstruktion von Gender-Kategorien sprechen, wüssten wir mehr über die Kontexte dieses Interviews. Trotz all dieser Deutungsmöglichkeiten scheint es mir sicherer, wenn wir uns im weiteren Fortgang unserer Vorlesung weniger mit unsicheren Epitexten und mehr mit den lyrischen Texten Alfonsina Stornis beschäftigen.

Wenige Jahre nach der (Rück-) Einwanderung nach Argentinien stand die ehedem finanziell gesicherte Familie mit leeren Händen da. Alle Investitionen in kleine Firmen oder Cafés waren gescheitert. Der Vater, der 1906 verstarb, entwickelte sich zum Alkoholiker und wurde zu einer traumatischen Gestalt für die kleine Alfonsina, die seit dem Alter von elf Jahren als Näherin in der Familie mitarbeiten und sich ihren Lebensunterhalt verdienen musste.

Immer wieder, auf eine fast obsessive Weise, hat Alfonsina Storni versucht, sich mit der Figur des verstorbenen Vaters literarisch und lyrisch auseinanderzusetzen. Neunzehn Jahre nach dessen Tod, also wohl im Jahr 1925, erschien ihr Gedicht De mi padre se cuenta. Es entstammt dem für die weitere Entwicklung der Lyrikerin enorm wichtigen Gedichtband Ocre, der einen Meilenstein in ihrer persönlichen wie literarischen Entwicklung markiert. Ein Blick auf diese Komposition genügt Ihnen, das Gedicht als Sonett zu identifizieren:

\footnotetext{
Von meinem Vater erzählt man, dass er ging auf die Jagd, Frühmorgens, wenn es tagte, von seinem Windhund gefolgt, Und auf dem langen Wege, sich ein wenig zu zerstreuen, Schaute er ihm in die Augen, und es winselte der Hund.

Dass er streifte durch Wälder und suchte eine Schlange, Die dreist, und fand er sie auf ihrem Schwanze erhoben Und bereit zum Angriff, schoss er mit frecher Kugel Lustvoll der Schlange den Kopf entzwei.
}

4 Vgl. Ette, Ottmar: Gender Trouble: José Martí and Juana Borrero. In: Font, Mauricio A. / Quiroz, Alfonso W. (Hg.): The Cuban Republic and José Martí. Reception and Use of a National Symbol. Lanham - Boulder - New York - Toronto - Oxford: Lexington Books 2006, S. 180-193 u. 230-233. 


\begin{abstract}
Dass er ganze Tage lang vagabundierend, umherstreifend, Nicht wieder nach Hause gekehrt, denn wie ein Einsiedler Nährt' er sich von Vögeln und schlief auf dem Boden.
\end{abstract}

Und alleine, wenn der Zonda große Massen an brennendem Sand und Insekten in den glühenden Wüsten San Juans Aufwirbelt, sang er und sang unter dem Himmel. ${ }^{5}$

Betrachten wir dieses klassische postmodernistische Sonett aus vierzehn Vierzehnsilbern näher, so fällt zunächst die klar zweigeteilte und in beiden Teilen wiederum halbierte Struktur auf. Dies wird bereits anhand der syntaktischen Strukturen deutlich, umfasst jede Strophe doch jeweils einen einzigen Satz, stimmen Strophen- und Satzgrenzen also miteinander überein. Bereits auf den ersten Blick erscheint das Sonett wie aus einem Guss gefertigt, wie es dem unveränderlichen Bild eines Vaters entspricht, aus Alfonsinas Sicht für die Ewigkeit gemacht.

Auf Ebene der Form ist das Reimschema des Sonetts $a b b a$, dann $c d c d$, in den Terzetten schließlich eef und ggf: eine klassische Form. Damit haben wir sowohl den umschlingenden Reim im ersten Quartett als auch den Kreuzreim im zweiten Quartett, danach einen Paarreim zu Beginn des ersten Terzetts und schließlich einen Paarreim zu Beginn des zweiten Terzetts, wobei wir die letzten vier Verse des Gedichts wiederum sehr wohl wie im ersten Quartett als umschlingenden Reim deuten dürfen. Durch dieses Reimschema bewegt sich das Gedicht mittels Variationen wieder zurück zum ursprünglichen umschlingenden Reim: Alfonsina Storni hat eine in sich ruhende Form mit ihren Variationen ohne gewagte formale Experimente gewählt.

Wir können auf formaler Ebene verschiedene Strukturen des Sonetts festhalten. Erstens beobachten wir auf der syntaktischen Ebene eine klare Vierteilung des Gedichts beziehungsweise eine doppelte Zweiteilung, indem Strophengrenzen und Satzgrenzen ausnahmslos übereinstimmen. Auf der grammatikalischen Ebene ergibt sich zweitens eine andere Struktur, in welcher die ersten drei Strophen als eine Konstruktion mit Nebensatz zusammengehören, was jeweils zu Beginn der zweiten und dritten Strophe durch das „Que“ angedeutet wird. Diese Verknüpfung fehlt aber in der vierten Strophe. Drittens ragt auf der Ebene des Reimschemas vor allem das zweite Quartett hervor durch das nun zum Kreuzreim überwechselnde Reimschema. Schon so ergibt sich eine recht komplexe Struktur der Verweisungszusammenhänge formaler Art.

5 Storni, Alfonsina: De mi padre se cuenta. In Villarino, María de (Hg.): Alfonsina Storni: Antología. Prólogo y selección de María de Villarino. Buenos Aires: Ediciones Culturales Argentinas 1961, S. 59. 
Im Zentrum des gesamten Gedichts steht die Figur des Vaters, die gleichsam aus der Distanz wahrgenommen wird: „se cuenta“, von ihr wird erzählt. Nichts erfahren wir über den Trunkenbold, nichts über die Gewalttätigkeiten, die das noch junge Mädchen traumatisierten. Die gewählte Distanz vergrößert sich noch, da der Vater zur Jagd aufbricht, also das Haus verlässt, was in der Tat zu einer Opposition und Echowirkung führt. Diese ergibt sich zwischen erstem Quartett und erstem Terzett, nämlich zwischen „caza“ und „casa“, was argentinisch ausgesprochen fürwahr keine Minimaldifferenz ergibt.

Das Unbegreifliche und Unfassbare wird im ersten Quartett an der Beziehung zwischen Herr und Hund, zwischen Mensch und Tier deutlich und greifbar; kommt es zwischen beiden zu einem direkten Blickkontakt, so ist dies für den Hund eine schmerzhafte und beunruhigende Erfahrung, beginnt er doch zu winseln oder $\mathrm{zu}$ stöhnen. Für den Vater hingegen ist dies Zeitvertreib, ein „divertirse“. Doch der Hund spürt die massive Gewalt, die von der Figur des Vaters ausgeht. Der Vater wird zusätzlich zur Erzähldistanz des lyrischen Ich, das nicht aus eigener Erfahrung berichten kann, an die Entfernung, an die langen Wege und die Situation einer problematischen Kommunikation angeschlossen, die nicht zum Sprachlichen vordringt, sondern im Vorsprachlichen verharrt. Die Sprache der Augen, das Sich-Anblicken von Herr und Hund sowie das Stöhnen des letzteren geben uns Aufschluss über ein verborgenes Inneres der Vaterfigur, welche das Sonett nicht negiert, aber auch nicht direkt zur Sprache bringt. Doch der Vater ist weit vom Haus, von seinem Zuhause, entfernt und überlässt sich ganz seinem „gozar“, seiner Lust.

Das zweite Quartett ist grammatikalisch parallel zum ersten aufgebaut, wobei es elliptisch an den Eingangsvers des Sonetts anschließt und diesen fortführt. Dass hierbei keine Einheit zwischen beiden Quartetten entstehen kann, ist - wie wir sahen - schon im Reimschema angezeigt, durch das eine größere Unruhe und eine Steigerung der Spannung in das Sonett gelangen. Im Zentrum dieses zweiten Quartetts steht wiederum eine Begegnung zwischen Mensch und Tier, diesmal zwischen dem Vater und der Schlange, die nicht etwa zufällig angetroffen, sondern gesucht und endlich gefunden wird. Sie erleidet einen geradezu ritualisierten Tod, der auf der Seite des Menschen und Mannes nun nicht mehr nur Zerstreuung, sondern weit mehr noch Lust (,gozaba“) bedeutet. Der Vater lebt in dieser erneuten Begegnung zwischen Tier und Mensch seine ganze angestaute Gewalt, seine ganze Lust in einem Akt gesuchter und gezielter Tötung aus.

Für das Tier ist in dieser Tier-Mensch-Beziehung eine Steigerung insoweit zu erkennen, als nun nicht länger nur das Leiden, sondern der Tod hereinbricht. Auch hier ist es der Kopf, wenn auch nicht die Augen, welche hiervon betroffen oder mehr noch getroffen werden. An die Stelle des Blickes ist dessen Materialisierung in der Gewehrkugel getreten: Die Sprache der Augen ist zur Sprache des 
Gewehrlaufs mutiert. Die Schlange, zum Angriff bereit, wird just in diesem Augenblick lustvoll und gezielt getötet - und es ist kein Zufall, dass „la serpiente“ im Gegensatz zu „el galgo“ weiblich ist.

Auch das erste Terzett schließt sich syntaktisch parallel zum zweiten an den ersten Vers des Gedichts an, so dass zwischen diesen drei Strophen eine enge Beziehung entsteht, aus welcher die letzte Strophe des zweiten Terzetts herausragen wird. In der dritten Strophe wird das räumliche Freisein, das Vagabundieren und Verlassen des Hauses auf unbestimmte Zeit, in den Mittelpunkt gerückt, eben jenes Vagabundieren, das wir im Gedicht Juana de Ibarbourous bereits als Merkmal männlichen Lebensstils kennengelernt hatten. Dem Lebensmodell des Jägers wird zugleich mittels eines Vergleichs das Lebensmodell des Eremiten an die Seite gestellt, des unabhängig von jeder Familie lebenden Einsiedlers, der sich selbst ernährt und in größter Bescheidenheit und Freiheit lebt, allein sich selbst verantwortlich.

Diese Freiheit und Unverantwortlichkeit wird dann in der letzten Strophe, im zweiten Terzett, gesteigert, eine Strophe, die zwar durch die Konjunktion „y“ an das Vorangehende angeschlossen ist, nicht länger aber an die fremde Rede. Denn jetzt heißt es nicht mehr „Que“ oder „Y que“: Die Rede wird vielmehr vom weiblichen lyrischen Ich selbst getragen. Das „sólo“ könnte in dieser Form oder auch ohne Akzent geschrieben werden, denn das Singen des Vaters alleine in der Wildnis außerhalb seines Zuhauses und unter freiem Himmel entwirft das starke Schlussbild eines Gedichts, in welchem der Vater für die Freiheiten seines Geschlechts und für jene Unabhängigkeit steht, von welcher die Frauen zuhause nur träumen könnten. Denn sie sind an das Haus („la casa“) gebunden, während der Mann auf die Jagd („la caza“) geht, tut, was ihm Lust verschafft, und unter freiem Himmel, nur sich selbst verantwortlich, schläft.

Im Zentrum des zweiten Terzetts steht wiederum die Kommunikation zum einen mit der Natur, mehr aber noch mit einem unbekannten Adressaten, wobei der Austausch nicht mehr in einer vorsprachlichen Form erfolgt, sondern sich in der Form des Gesangs vollzieht. Dieser einsame Gesang steht der Lyra, steht dem lyrischen Gesang sehr nahe und macht auf Parallelen mit dem weiblichen Ich aufmerksam, die gewiss zunächst nicht in den Blick gerückt wären. Es ist ein einsames Singen, das an diesem Punkt erstmals geographisch verortet wird in den „desiertos sanjuaninos“, also in den weiten menschenleeren Trockengebieten um die Provinzhauptstadt San Juan, wo die Familie einst gelebt hatte. Zugleich ist es eine Szenerie der Naturgewalten, in der ein starker, von den Anden kommender Wind sowohl die belebte als auch die unbelebte Natur aufwirbelt und als Partikel durch die glühende Luft schleudert.

Dabei handelt es sich um eine Situation der Grenze, im „desierto“, in welchem der Mensch an die Anökumene stößt. Diese Siedlungsgrenze am Rande der 
Ökumene, der dauerhaften menschlichen Besiedlung - das macht eine intensivere Beschäftigung mit der argentinischen Literatur klar -, ist im 19. Jahrhundert nicht nur die zur Natur, sondern auch zu anderen nomadisierenden Lebensformen indianischer Kulturen, von denen hier jedoch noch nicht einmal mehr Spuren vorhanden sind. Es ist vielmehr der nomadisierende Weiße, der nun seinerseits die Wüste betritt, um eine außermenschliche Kommunikation zu suchen, zu der es kein direktes Objekt, sondern nur Zeit- und Ortsbestimmungen gibt. Lange vorbei sind die Zeiten, in denen ein Esteban Echeverría in La Cautiva die Welt der indigenen Stämme als den Weißen gefährliche Macht besang.

Was das Reimschema bereits andeutete, bestätigt das Gedicht auf seiner semantischen Ebene. Der Vater ist dem lyrischen Ich sicherlich nicht näher gekommen, die Distanz, die vom ersten Vers an bestand, scheint unüberbrückbar: Es ist, als lebte der Vater in einer eigenen Welt, getrennt von Familie und Zuhause. Die Figur des Vaters bleibt distanziert, erscheint als gewalttätig, todbringend, letztlich rätselhaft, unergründlich, durchaus aber nicht hassenswert. Dieser Figur kommen Attribute zu, die für die weibliche Autorin durchaus erstrebenswert waren und die sie in ihrem Leben als Frau, an entsprechende GenderNormen gefesselt, vermisste.

Der Vater ist kein Ausgestoßener, verlässt er doch freiwillig das Haus, zu dem er ja gewiss wieder nach eigenem Gutdünken und Entschluss zurückkehren wird. Und doch ist er der Andere, dem nicht der geordnete Raum des Hauses, sondern die offenen Räume zugeordnet sind und offenstehen. Die Gewalttätigkeit erscheint vor allem im zweiten Quartett beim Töten der Schlange, das sich schon durch die Zischlaute der umgebenden Verse andeutete, welche hier wesentlich häufiger sind als in der vorangegangenen Strophe. Das zischelnde „,“ kommt freilich wieder im letzten Vers des ersten Terzetts, in welchem ebenfalls der Tod der Vögel eine Rolle spielt, handelt es sich dabei auch um eine das eigene Leben erhaltende Jagd, in welcher das Tier zum Lebensmittel wird: „Se alimentaba de aves“, „Nährt' er sich von Vögeln“. In diesem Vers erfolgt das Töten, anders als im Falle der Schlange, nicht aus purer Lust.

Auf der zeitlichen Ebene ist alles distanziert, dominiert das „imperfecto“, das in der Zeitenfolge den Vater quasi noch mehr entrückt und die Bewegungslosigkeit der Szenerie wie in einem Gemälde oder mehr noch einem langsam, einem quälend langsam und fast traumatisch ablaufenden Film betont. Die Gestalt des Vaters ist in diesem Gedicht Alfonsina Stornis genau das: unergründlich und traumatisch, unnahbar und unfassbar, und doch irgendwie faszinierend und frei. Kein Weg führt zu ihr, und doch führt auch kein weg von dieser Vaterfigur weg.

Dies wird deutlich, wenn wir auf der kotextuellen Ebene in Stornis Band Ocre nach dem innerfamiliären Pendant suchen und auf ein Gedicht stoßen, das der Figur der Mutter gewidmet ist. Erlauben Sie mithin ein zweites Gedicht aus 
dem für Alfonsina Stornis Entwicklung so wichtigen Gedichtband aus dem Jahr 1925. Auch hier, das wird Sie aus Gründen des Gleichgewichts nicht überraschen, handelt es sich um ein Sonett, das sich dieses Mal aber um die Mutter bemüht:

\footnotetext{
Nicht die großen Wahrheiten frag' ich Dich, zumal

Du sie niemals beantwortest; ich dring nur in Dich, Ob, als Du mich trugst, der Mond Zeuge war,

Spazierend durch dunkle Innenhöfe in Blüte.

Und ob ich, an Deinem Busen lateinischer Inbrunst Schlummernd hörte, wie ein raues Meer aus Klängen Dich einlullte in den Nächten und Du schautest im Gold Der Dämmerung, wie all die Seevögel versanken.

Denn meine Seele ist gänzlich entrückt und reisend, Und es umhüllt sie eine Wolke leichten Wahns, Wenn der Neumond am bläulichen Himmel aufsteigt.

Und wenn's Meer seine starken Räucherkammern öffnet, Wiegend im klingenden Singen der Seeleute, Schauen magst die großen Vögel im ziellosen Flug. ${ }^{6}$
}

In diesem Gedicht finden wir, kontrastiv zur Figur des Vaters, jene der Mutter, aus großer körperlicher Nähe betrachtet. Grammatikalisch zeigt sich dies bereits im ersten Vers, wo das lyrische Ich in unmittelbare Nähe zum „te“ als Objekt seiner Frage kommt, also eine direkte Kommunikationssituation herstellt, zu der es mit dem Vater niemals kam. Überdies ist das lyrische Ich eine Frucht dieser Mutter, die es in sich trug, ist deren Kind und fragt nach den Kontexten vor der Geburt, ja nach der Zeugenschaft des Mondes, der zweimal im Sonett auftaucht. Es ist diese Welt der weiblichen Symbole, welche das Sonett gestalten und begleiten. Kein Mann scheint am Zeugungsprozess je beteiligt gewesen zu sein: Alles scheint sich in der Beziehung zwischen Mutter, Ich und Mond (,la luna“, dem weiblichen Mond oder der Möndin) abgespielt zu haben. Dabei ist im ersten Quartett der Raum eng begrenzt: Ein Haus mit seinem Innenhof erscheint, ein Patio, das wohl den Zutritt des Mondes, nicht aber die Öffnung zum Draußen erlaubt. Diese Intimität wird durch die Pflanzenmetaphorik der Blüte (,la flor“) in etwas Organisches gewendet.

Dieser Raum wird im zweiten Quartett noch weiter verengt auf den KörperLeib der Mutter, an deren Busen und in deren Schoße das Kind ruht und schläft.

6 Storni, Alfonsina: Palabra a mi Madre. In Villarino, María de (Hg.): Alfonsina Storni, S. 59 f. 
Dies ist eine Verbindung zwischen Körper und Körper, die gänzlich andere Beziehungen herstellt zwischen dem weiblichen Ich und der Mutter als zwischen dem Ich und dem fernen Vater im vorigen Sonett. Gerade dadurch, dass Alfonsina Storni beide Sonette aus ihrem Band Ocre mit einer Reihe formaler Parallelen ausstattet - so etwa die Übereinstimmung von Strophengrenzen und Satzgrenzen -, treten die Unterschiede zwischen dem Portrait des Vaters und dem Portrait der Mutter kotextuell, also im selben Band, umso deutlicher und schärfer hervor.

Hatte im ersten Quartett der Mond für die notwendige Beleuchtung gesorgt, so wiegt im zweiten Quartett - in ähnlicher Symbolik wie bei Gabriela Mistral bei wohl nur dunklem Licht das Meer Mutter und Kind in den Schlaf. Allein aus der Perspektive der Mutter erscheint am Horizont und in weiter Entfernung eine andere Welt, die der Meeresvögel, die im Sonnenuntergang sich auf den Weg machen und im Meer versinken. Dieses Bild der Vögel, welche ohne Zielort ihren Flug unternehmen, wird am Ende, im letzten Vers des zweiten Terzetts, wiederkehren und in den engen Bereich der Häuslichkeit der Mutter jene Weite einführen, der sich ihre Tochter, „viajera“ in der wichtigen Versendstellung, verpflichtet weiß. Das Meer führt nicht nur die Ebene des Wiegens und Klanges, sondern auch der Weite herbei, welche am Ende des zweiten Quartettes nun zu den Terzetten und damit zu einer anderen Welt überleitet.

Wieder werden - wie bereits erwähnt - die vier Strophen von vier Sätzen gebildet, wenn hier auch die elliptische Struktur fehlt, mit der alles an eine fremde Rede angebunden war. Im Sonett an die Mutter ist es die direkte körperliche Kommunikation zwischen Mutter und Kind, welche Handlung und Semantik vorantreibt. Die große Geborgenheit und schläfrige Ruhe mag schon daran anschaulich werden, dass wir es in beiden Quartetten mit umschlingendem Reim zu tun haben, dass wir also eine größere Statik, eine geringere Unruhe in den beiden ersten Quartetten beobachten können. Insofern erhalten auch die letzten vier Verse des Sonetts nach dem Paarreim des ersten Terzetts eine Situation mehr aufrecht, als dass sie zu ihr zurückkehrten. Das Gedicht strahlt schon auf der formalen Ebene eine große Ruhe aus, welche freilich auf der semantischen Ebene immer wieder mit Verweisen auf die Weite und auf den Gesang der männlichen Seeleute durchbrochen wird.

Freilich führt gerade der Beginn des ersten Terzetts ein starkes Moment der Unruhe ins Feld, welches auf die Tochter, auf das lyrische Ich, bezogen ist. Denn diese Tochter ist „fantástica“ und „viajera“, von einer „nube de locura ligera“ umhüllt: Sie fahndet nach jenen Elementen wie Mond, Meer und Weite, nach dem Flug der Vögel und dem Gesang der Seeleute, welche sie bereits im Bauch der Mutter verändert und auf eine Welt fernab der Bezogenheit auf die Innenhöfe und das Haus orientiert haben könnten. Die Reisen, aber auch der Wahnsinn klopfen 
an die Türe der innerlichen Häuslichkeit: Eine Welt des Fantastischen, der Entrückung und der Fantasie blitzen in Alfonsina Stornis Sonett auf.

Insofern durchbrechen die Terzette in gewisser Weise die Ruhe und Abgeschlossenheit der beiden Quartette, welche die Exposition lieferten, denen nun die etwas beunruhigende Durchführung folgt. Es geht um die Seele des lyrischen Ich, welche sich den ruhigen und intimen, für Frauen in der damaligen Gesellschaft als adäquat erachteten Bereichen entzieht und buchstäblich das Weite sucht. Woher aber rührt dieser leichte Wahnsinn, diese - um es mit einem anderen Wort zu sagen - Entrückung? Woher rührt dieses unbedingte Suchen nach Bewegung, nach einer Mobilität ohne eigentliches Ziel?

In beiden Attributen können wir schon jetzt - obwohl das Gedicht kotextuell jenem an den Vater gerichteten Sonett vorausgeht - Elemente der rastlosen, am Draußen ausgerichteten und oft unter freiem Himmel lebenden Vaterfigur erkennen. Das zweite Terzett, das mit den Räucherpfannen oder -kammern des Meeres beginnt, setzt wie im Gedicht über den Vater mit der Konjunktion „y“ ein, ist aber erneut ganz dem eigenen Ich gewidmet, so dass deutlich die Gewichte von der Mutter zum Ich in den Terzetten verschoben werden. In viel stärkerem Maße als im Gedicht an den Vater ist im Gedicht an die Mutter vom weiblichen Ich, von der Tochter, Die Rede. Die Identifikation mit und Abgrenzung von der Mutter sorgt für eine wesentlich stärkere Präsenz des weiblichen Ich.

Erneut taucht im Sonett an die Mutter ein Singen auf, ein Singen von Männerstimmen, welche freilich als Seeleute dem Meer verpflichtet sind; und erneut sind hier ebenfalls die Vögel präsent, die freilich nicht zum Jagdgegenstand des Vaters werden, sondern Objekte der sehnsüchtigen Blicke des lyrischen Ich sind, die in ihnen ,aves que pasan sin destino“, ziellose Vögel, zu erkennen glauben. So entwickelt sich das Gedicht von einer sehr eingeengten Räumlichkeit und Zeitlichkeit zu einer absoluten Offenheit, in welcher sowohl die Räume als auch die Träume, die Zeiten und insbesondere die Zukunft, das Schicksal, offen sind.

Gewiss sind es nicht zuletzt autobiographische Elemente, die etwa in den „fervores latinos“ auf die europäische Herkunft der Mutter, aber auch der eigenen Geburt in der italienischen Schweiz anspielen. Das väterliche Element ist nahezu abwesend, wären hier nicht die Gesänge der Seeleute, der Männer, die an den Vater zumindest kotextuell erinnern und dessen Singen unter dem Himmel beschwören. Sicherlich war auch die Mutter im Gedicht an den Vater abwesend. Doch sind es im Sonett an die Mutter die Attribute des Vaters, des Mannes, die auf das lyrische Ich übergehen, das von jenen großen Fragen gequält wird, von jener Entgrenzung der „locura“, von der es schon im allerersten Vers des Gedichts weiß, dass die Mutter darauf keine Antworten geben kann. So bleibt der unhörbare Dialog mit dem Vater, der stets ferne ist, dessen Gestalt nicht erscheint und dessen Figur - so glaubt man zumindest in der kotextuellen Lektüre zu erken- 
nen - dennoch allgegenwärtig ist; und sei es als Frage, als Freiheit oder als Fantasie in der Wolke leichten Wahnsinns.

Ich kann nur schwer der Versuchung widerstehen, Ihnen nun auch noch ein drittes Gedicht, und zwar das Eröffnungsgedicht des Bandes Ocre, im Rahmen unserer Vorlesung vorzustellen. Es trägt den Titel Humildad, also die lateinische „humilitas“, und es ist auch in einer noch genauer zu ergründenden Weise einer Vatergestalt gewidmet. Erneut handelt es sich um ein Sonett, und so will ich es beim Zitat einiger weniger Verse belassen, um Alfonsina Storni auch in anderen Gedichtformen zu präsentieren. Die ersten beiden Verse des Sonetts lauten: „Yo he sido aquella que paseó orgullosa / El oro falso de unas cuantas rimas“ - „Ich bin die, die einst stolz / Durch's falsche Gold einiger Verse“. Das liest sich aus heutiger Perspektive reichlich beliebig, fehlt uns heute doch der literaturgeschichtliche Hintergrund, auf den sich diese Verse beziehen. Was können wir zur Aufklärung dieses Sachverhalts beitragen?

Nun, für die damaligen Leserinnen und Leser konnte nicht der geringste Zweifel bestehen: Dieses Sonett Humildad thematisierte nicht nur eine ästhetische Wende im Schaffen der Alfonsina Storni. Zugleich wurde auf eine weitere ästhetische Wende im Schaffen eines großen Dichters angespielt, dessen Eingangsvers in den Cantos de vida y esperanza lautete: „Yo soy aquel que ayer no más decía / El verso azul y la canción profana / En cuya noche un ruiseñor había / Que era alondra de luz por la mañana.“

Gerade einmal zwanzig Jahre war es her, dass diese großen Verse im Jahr 1905 eine Art Selbstabrechnung und Affirmation des eigenen lyrischen Ich im Schaffen des großen Nikaraguaners Rubén Darío signalisierten und einen der wichtigen Wendepunkte innerhalb der modernistischen Ästhetik mit sich brachten. 1925 griff Alfonsina Storni auf diese einem lateinamerikanischen Lesepublikum altbekannten Verse zurück, um wesentlich ,humilder‘ auf ihre eigene Entwicklung einzugehen, Selbstüberschätzungen vorzubeugen, aber auch dem modernistischen Übervater Rubén Darío eine Absage zu erteilen und ihrer eigenen weiblichen Subjektivität gerade an dieser Stelle ein kleines literarisches Denkmal zu setzen. Auch hieran erkennen wir: Für die Generation schreibender Frauen, für die Lyrikerinnen der ersten Hälfte unseres Jahrhunderts war der hispanoamerikanische Modernismo keineswegs eine tote, zur Literaturgeschichte gewordene Bewegung, sondern noch immer Herausforderung und Stachel innerhalb dessen, was wir mit postmodernistischer Lyrik meinen. Denn gegenüber Darío bedeuteten diese Verse keinen avantgardistischen Bruch, sondern Selbstaffirmation als lateinamerikanische Dichterin, die in einer nachdarianischen Zeit - Rubén Darío war 1916 verstorben - neue Perspektiven für den Modernismo im Postmodernismo eröffnete.

Lassen Sie uns ein letztes Mal zum Thema des Vaters zurückkehren mit einem weiteren Gedicht aus dem gerade für die postmodernistische Dichtung so wichti- 
gen Gedichtband Ocre: der Romance de la Venganza! Ich kann dieses Gedicht hier freilich nur in Teilen wiedergeben. Es knüpft überdeutlich an das Bild des Vaters an, wie es in De mi padre se cuenta entwickelt wurde und darf daher nicht ganz aus unserer Betrachtung Alfonsina Stornis verschwinden:

\author{
Hochgewachsner und so schöner Jäger, \\ Auf der Erde gibt's nicht zwei, \\ Ging zur Jagd am Nachmittage, \\ In den Bergen unseres Herrn. \\ [...] \\ Da er kehrt zurück, so sang er \\ Sanft und sacht mit leisem Ton, \\ Von dem Baum herab verschlungen \\ Blickt' ihn eine Schlange an.
}

\begin{abstract}
All die Vögel wollt' sie rächen, Doch der Jäger gewaltig war, Mit der Kling' aus festem Stahle Hieb er der Schlange ab den Kopf.

Doch geduldig auf ihn wartete Ich, ganz wenige Schritte weg ... Fesselt' ihn mit meiner Haarpracht Und beherrschte seine Wut.

[...]

Nicht mit Waffen ich ihn getötet, Sucht' ich doch 'nen schlimmern Tod: So küsst' ich ihn so süß und sachte, Dass sein Herz ich bersten ließ! $[\ldots]^{7}$
\end{abstract}

Wir erkennen in diesem Gedicht ohne größere Schwierigkeiten - und Eyda Machin ${ }^{8}$ hat dies in ihrem Aufsatz bereits getan - die übereinstimmenden Elemente, die bei den offensichtlichen Lexemrekurrenzen und symbolhaften Menschen- und Tiergestalten beginnen, zugleich aber eine andere Inszenierungsart und einen anderen Handlungsablauf aufweisen. Denn in diesem Gedicht, das im selben Gedichtband kotextuelle Bezüge zu ihrem dem Vater gewidmeten Sonett aufweist, kommt als zusätzliche Kommunikationsebene die zwischen Mensch

7 Storni, Alfonsina: Romance de la Venganza. In Bast Glas, Cristina / Cardona, Francesc (Hg.): Alfonsina Storni. Antalogía poética. Barcelona: Ediciones Brontes 2014, S. $116 \mathrm{f}$.

8 Vgl. Machin, Eyda: La mujer y la escritura: Juana de Ibarbourou y Alfonsina Storni. In: Heydenreich, Titus (Hg.): Der Umgang mit dem Fremden. München: Fink 1986, S. 65-90. 
und Mensch, zwischen Jäger und Mädchen, zwischen Mann und Frau hinzu. Ja es geht noch weit darüber hinaus: Der schöne Jäger, der die Tiere tötet, wird selbst zum Gejagten, zum Opfer der Frau. Denn sie verwendet die Waffen einer Frau, denen er nichts entgegenzusetzen hat.

In diesem Gedicht ist die Distanz, welche die Beziehungen im Sonett noch charakterisierte, überbrückt und zugleich die Grausamkeit in das lyrische Ich übernommen: Die junge Frau mit ihren langen Haaren übt Rache, süße Rache an diesem Mann. Im Gedicht wird nicht klar, wofür sie Rache übt - die Schlange hätte Gründe gehabt, die Tiere zu rächen - aber die Frau? Die Rache erfolgt nicht aus der Position der Tochter, sondern aus jener der liebenden Frau, ja der Geliebten, die ihren Liebespartner gleichzeitig hasst und einer grausamen Tötungsart unterzieht. Dem Einfangen des Mannes folgt die Zerstörung von innen, die Zersprengung seines Herzens, gegen die er sich nicht zu wehren vermag.

Es ist ohne Zweifel leicht möglich, diese Deutungsart autobiographisch zu untermauern und psychoanalytisch zu interpretieren: Die Obsession der längst verstorbenen Vaterfigur ist in den Gedichten Alfonsina Stornis so überdeutlich, dass sich in der Tat ein „Mythe Personnel“ (im Sinne des französischen Literaturtheoretikers, Psychoanalytikers und Dichters Charles Mauron ${ }^{9}$ ) im Kontext eines ganzen Netzwerks von Obsessionen und wiederkehrenden traumatischen Situationen ausmachen lässt. Ich kann Ihnen diese These leider nicht im Rahmen unserer Vorlesung belegen, müsste ich doch noch weitere Gedichte und auch die essayistische Produktion von Alfonsina Storni vorführen, wozu uns leider der Platz fehlt. Aber welche prägende Bedeutung der schöne Vater und Jäger im Werk wie im Denken der argentinischen Dichterin besitzt, haben Sie sicherlich anhand der angeführten Gedichte ermessen können.

Allerdings scheint mir die ästhetische Dimension nicht weniger wichtig zu sein, greift Alfonsina Storni in diesem Gedicht doch nicht auf die Form des Sonetts zurück, sondern auf den Romance, eine traditionelle Form des populären Achtsilbers, den auch die Modernisten sehr gerne in ihrer lyrischen Produktion benutzten, unter ihnen vor allem José Martí, der es hierin zu einer unbestrittenen Meisterschaft brachte. Damit greift Storni auf eine fruchtbare Traditionslinie zurück, die nicht nach Frankreich (wie bei Rubén Darío), sondern nach Spanien führt und eine ganze Filiation hispanoamerikanischer Lyrik begründete. Diese populäre Form hatte gerade im letzten Drittel des 19. Jahrhunderts die höheren ästhetischen Weihen erhalten und neue Anwendungsformen dichterischer Praxis gefunden.

9 Vgl. Mauron, Charles: Des Métaphores obsédantes au Mythe Personnel. Paris: José Corti 1995. 
Mit ihrem Rückgriff auf eine aus der Volkslyrik stammende Form, die von den Modernisten in der Tradition Martís und seiner Versos sencillos aufgewertet wurde, bezieht sich Alfonsina Storni auf eine Filiation, welche sich dem überladenen Zwang modernistischer Schreibweisen in der Nachfolge Daríos zu entziehen suchte. Zugleich transponierte sie aber das Geschehen in eine Welt mythischer Schlichtheit, Einfachheit und Einheit, welche die mittlerweile überwundene Distanz zum Vater und die Preisgabe ihrer Liebe in einem gegenläufigen Verfahren mit gewollter Brutalität wieder in das Reich von Fabeln und Legenden verbannte. Die Spur der Arbeit an dieser obsessiven Konstellation ist überdeutlich und leicht an den auffälligen Lexem-Rekurrenzen erkennbar.

Sicherlich ließe sich dieses Gedicht auch autobiographisch auf die unglückliche Liebesbeziehung zu einem hochgestellten, um vierundzwanzig Jahre älteren Mann in Santa Fé beziehen, eine Verbindung, welche die junge Alfonsina Storni niemals öffentlich machen konnte und die sie teuer bezahlen musste. Die herrschenden Geschlechterverhältnisse trafen sie mit voller Wucht, eine befriedigende Lösung konnte nicht gefunden werden: Sie wurde ein Opfer der geschlechterspezifischen Ungleichheiten.

Doch längst war sie zu einer ausdrucksstarken Lyrikerin geworden, die viel von diesem Mann, viel aber auch von ihrer schreibenden Mutter übernommen hatte, mit der sie 1905, also im Alter von dreizehn Jahren, zum ersten Mal bei einer Lesung öffentlich aufgetreten war. Auf keinen Fall aber wollte Alfonsina die Rolle ihrer Mutter übernehmen, die nach ihrer Ansicht niemals ihre Freiheit hatte finden und durchsetzen können. Die Tochter indes besaß genügend Durchsetzungsvermögen, um ihren Weg auch alleine gehen zu können. Die frühen Gedichte der Storni geben darüber Auskunft, wie schwer es ihr fiel, sich von der traditionellen Frauenrolle loszusagen und diesen ganz eigenen Weg in den Feminismus als Schriftstellerin zu finden.

Alfonsina Storni besaß den Mut, im damaligen Argentinien eine alleinerziehende Mutter zu sein und sich ihrem immer stärker positionierenden Schreiben zu widmen. Sie entwickelte in der Folge eine Reihe politischer Aktivitäten, die sie als klare Feministin präsentieren. 1910 wurde sie Mitglied im „Comité Feminista de Santa Fé“ und schließlich auch dessen Vizepräsidentin. In einer Veränderung der dominanten Gesetzeslage erblickte sie die Grundlage für die Gleichstellung der Frauen in Gesellschaft und Alltagsleben. Macht sich, so Eyda Machín kritisch, bisweilen auch ein gewisser Fatalismus bei ihr breit bezüglich einer Ankettung der Frauen durch ihr Geschlecht, ${ }^{10}$ so sind doch immer wieder

10 Vgl. Machin, Eyda: La mujer y la escritura: Juana de Ibarbourou y Alfonsina Storni. In: Heydenreich, Titus (Hg.): Der Umgang mit dem Fremden. München: Fink 1986, S. 65-90. 
Kampf und Engagement der Storni überzeugend und bewundernswert. Durch dieses Engagement wurde sie zu einer Frauenfigur, die noch heute für viele Modellcharakter besitzt.

Ich möchte daher mit einem Gedicht unsere Beschäftigung mit der argentinischen Lyrikerin beenden, in welchem schon früh dieser Mut und Kampfeswille zum Ausdruck kam. Es handelt sich um ein dem Gedichtband El Dulce Daño entnommenes Poem, das in einem gewissen Gegensatz zu den Liebesgedichten dieser Sammlung von 1918 steht - eine Sammlung, die also ein Jahr vor Juana de Ibarbourous Las lenguas de diamante und César Vallejos Los heraldos negros erschien:

Du willst mich hell, Willst mich aus Schaum, Willst mich aus Perlmutt. Dass ich weiß wie die Lilie Über alle, und keusch.

Mit feinem Duft.

Geschlossne Blumenkrone.

Kein Strahl des Mondes Dürft' mich berühren. Keine einzge Margerite Sage mir Schwester. Du willst mich Schnee, Du willst mich weiß, Du willst mich hell.

Du, der Du alle Kelch' in Deiner Hand, Von Früchten und Honig Die Lippen blutrot, $\mathrm{Du}$, beim Bankett Mit Weintrieben bedeckt Die Fleischstücke lässt, Deinen Bacchus ehrst, $\mathrm{Du}$, der Du in den schwarzen Gärten der Täuschung Ganz in Rot gehüllt Zur Verwüstung ranntest. Du, der Du das Skelett Intakt noch bewahrest, Noch weiß ich's nicht Durch welche Mirakel, Du forderst mich weiß, (Gott mag's Dir vergeben) 
Du forderst mich keusch,

(Gott mag's Dir vergeben)

Du forderst mich hell!

Flieh hin zu den Wäldern;

Verschwind' in die Berge,

Putz Dir den Mund ab;

Leb' in den Hütten;

Berühr' mit den Händen

Die durchnässte Erde;

Nähr' Deinen Körper

Mit bitteren Wurzeln;

Trink aus den Felsen;

Schlaf auf dem Frost;

Erneuer die Gewebe

Mit Schwefel und Wasser;

Sprich mit den Vögeln

Und wach' in Morgenhelle.

Und wenn Dein Fleisch

Dir sei genommen,

Und wenn Du darauf

Gesetzt die Seele,

Die in den Betten

Sich so sehr verheddert,

Dann, guter Mann,

Fordere' mich weiß,

Fordere mich Schnee,

Fordere mich keusch. ${ }^{11}$

Auch dieses Gedicht von Alfonsina Storni ist von einer im Vergleich mit der modernistischen Lyrik der Rubén Darío-Epigonen so überraschenden, ja schockierenden Einfachheit, dass es fast etwas schwer fällt, es einer längeren, eingehenderen Interpretation zu unterziehen. $\mathrm{Zu}$ deutlich sind die Partner voneinander abgegrenzt, zu klar werden die Geschlechterunterschiede benannt, zu erkennbar haben wir es mit Männer- und Frauenrollen zu tun, zu scharf wird in der Figur des Mannes die Doppelbödigkeit der zeitgenössischen Geschlechterkonstruktionen sichtbar. Sie erlauben dem Mann in der Gesellschaft alle Arten von Festen und Ausschweifungen, fesseln die Frau aber an ganz bestimmte enge Normen bezüglich Schönheit, sexueller Disponibilität und seelischer Vereinsamung.

11 Storni, Alfonsina: Tú me quieres blanca. In Bast Glas, Cristina / Cardona, Francesc (Hg.): Alfonsina Storni. Antalogía poética, S. $63 \mathrm{f}$. 
Gleichzeitig wird der Stolz des weiblichen Ich fühlbar, das dem Mann und dessen Anspruchsverständnis in offener Herausforderung entschlossen entgegentritt.

Derlei Konstruktionen und Ansprüche von männlicher, patriarchalischer Seite werden in diesem Gedicht mit eindeutiger Geste zurückgewiesen in einer literarischen Form, die sich zweifellos wegen ihrer Einfachheit und Schlichtheit auch für publizistische Zwecke und für den Gesang, für die musikalische Vertonung eignet. Tatsächlich sind eine ganze Reihe von Gedichten Alfonsina Stornis in die Geschichte der lateinamerikanischen „Canción“ eingegangen.

Die Frau wird zu Beginn des Gedichts durchgängig in Außensicht, als eine rein patriarchalische Konstruktion der Männergesellschaft mit ihren Ansprüchen und Forderungen gesehen, die gleichsam als Modell aus der Hand ,ihres ' Mannes präsentiert wird. Dieser aber wird zunehmend seines Diskurses entmachtet, ja schließlich einem Reinigungsprozess unterworfen, welcher - so scheint mir - seinerseits nicht unproblematisch ist. Denn die im weiteren Verlauf des Gedichts, bis kurz vor Schluss, angelegten Kriterien sind in der Tat eines Einsiedlers und Eremiten würdig: Eine Isotopie christlicher Religiosität wird in Fortsetzung der uns bereits bekannten Lexik aufgerufen, die verschiedentlich anhand symbolischer Handlungen im Gedicht benannt wird. Der Mann möge sich erst einmal selbst reinigen und zurücknehmen, bevor er die Frau mit seinen geschlechterspezifischen Ansprüchen - die wir als phallogozentrisch bezeichnen dürfen - konfrontiert. Nicht umsonst wird Gott angerufen zur Verzeihung der Prätentionen des Mannes, nicht umsonst entspringen die an die Frau herangetragenen Wertvorstellungen und Normen einer christlich-patriarchalischen Tradition, gegen die sich das lyrische Ich stemmt; und nicht umsonst behält die Stimme des weiblichen Ich klar die Oberhand, als es den Mann ins Gebirge oder in die Wüste schickt. Dass eine derartige Lyrik anders als die der Juana de Ibarbourou - die durchaus bestimmte feministische Forderungen wie die nach Rechtsgleichheit mittrug - dem männlichen Lesepublikum weniger gefiel, mag man verstehen können.

Wir finden im Gedicht Tú me quieres blanca jene Symbole wieder, die wir nun schon mehrfach in der Lyrik Alfonsina Stornis ausgemacht hatten. So taucht etwa der Mond auf, der hier gleichsam ausgesperrt werden soll; oder wir sehen Wälder und Berge, in die der Mann aufbrechen muss; wir erkennen die einfachen Hütten, aber auch die Einsamkeit und den feuchten Boden, Attribute, die dem Bild des Vaters als Jäger nicht fern sind, wie wir dies im ersten Gedicht über den Vater nachgezeichnet hatten. Und auch die Vögel dürfen nicht fehlen, welche nicht getötet werden, sondern mit denen eine nicht mehr nur vorsprachliche Kommunikation gleichsam in franziskanischer Manier aufgebaut werden soll. Auf diese Weise erscheint ein positives, gereinigtes Bild des Vaters, dem das männliche Pendant nachzueifern hat, um überhaupt einem Manns-Bild zu entsprechen, welches das weibliche Ich mit Ansprüchen konfrontieren dürfte. Es ist 
schon erstaunlich, wie präsent immer wieder die ambivalente Figur des Vaters in vielen Gedichten erscheint.

Zweifellos gehört dieses Gedicht nicht zu den ästhetisch gelungensten der argentinischen Dichterin; und zweifellos lässt sich an ihm auch nicht jene komplexe Reflexion der eigenen Rolle als Frau und Lyrikerin aufweisen, wie dies in anderen Gedichten bei voller Polysemie möglich war. Doch Tú me quieres blanca wirkt durch seine ungeheure Kraft und Entschlossenheit, mit der es dem patriarchalischen Diskurs offen zu Leibe rückt und die Männerrollen auf ihre Doppelbödigkeit hin analysiert. Es wird, in dieser so einfachen Form, die Problematik jener Geschlechterkonstruktionen deutlich, die keineswegs nur die Frau als Modell-Objekt des Mannes, sondern auch den Mann als ein Objekt der Frau darstellt. Denn in den Forderungen des weiblichen Ich ist ebenfalls ein Männerideal verborgen, das sehr wohl als Anspruch an den Mann ausformuliert wird. Dem Frauen-Bild des Mannes wird ein Männer-Bild der Frau gegenübergestellt.

Dabei ist klar, dass diese Umkehrung letztlich kein Umsturz und keine Verwirrung der Geschlechter, sondern lediglich eine Umkehrung und Umpolung ist, welche die Pole als solche beibehält, ja sogar zu stützen vermag. Von dieser Position aus ist es noch ein weiter Weg zu jenen Aussagen, die wir zu Beginn unserer Beschäftigung mit Alfonsina Storni festgehalten hatten. Es wäre daher verfehlt, in der argentinischen Dichterin lediglich die Feministin zu erblicken, jene Frau, die eine klare Trennlinie zwischen Mann und Frau zog und mit guten Gründen auf der politischen und juristischen Gleichstellung ihrer Geschlechtsgenossinnen beharrte.

Wieviel komplexer die lyrische Welt der Alfonsina Storni ist, haben unsere vorgängigen Gedichtinterpretationen gezeigt. Und doch ist es dieser literarische Bewusstwerdungsprozess, der bis heute das ästhetische wie soziale Vermächtnis der Storni nicht nur wachgehalten hat, sondern noch immer weiter verstärkt. Alfonsina Storni ist damit nicht jenem Vergessen anheimgefallen, das sie sich selbst prophezeite in ihrem bereits anzitierten Eingangsgedicht von Ocre, das mit den folgenden Versen endet: „De un cansado soplido / Me aventará al olvido“ („Von einem ermüdeten Wehen / Fächelt mir Luft zu das Vergessen“).

Diesem Vergessen ist Alfonsina Stornis Leben und Schaffen nicht überantwortet worden. Allerdings wäre es nötig, die Interpretation ihrer postmodernistischen Lyrik weniger eng an der autobiographischen Gängelrute zu halten. Dann könnten wir die ersten beiden Verse ihres Gedichtbandes El Dulce Daño einlösen, das den (wie oft bei der Storni) selbstaffirmativen Titel Así (So) trägt: „Hice el libro así: / Gimiendo, llorando, soñando, ay de mí“ („So machte ich das Buch: / Stöhnend, weinend, träumend, weh mir“).

Diese Suche nach Unmittelbarkeit, dieses Betonen des Eigenen, des NichtÜbernommenen, erinnert stark an die Verse José Martís, der ebenfalls auf dem 
So-Sein seiner Gedichte und Verse beharrte: „a nadie los pedí prestados“ (,Von niemandem wollte ich sie mir leihen“). Die weibliche Lyrik des Postmodernismo verdankt dem Schaffen des unermüdlichen Kubaners von Gabriela Mistral über Juana de Ibarbourou bis hin zu Alfonsina Storni gewiss sehr viel, entfaltete aber einen Ton, einen Klang der Authentizität und der Überzeugung, wie er innerhalb der Literaturentwicklung der lateinamerikanischen Länder unerhört und höchst eigenständig war. Es ist eine Lyrik, deren Ausstrahlung bis weit in unsere Gegenwart weist und eine literarische Tradition begründet, welche nicht allein die zeitgenössischen Lyrikerinnen in spanischer Sprache bis heute fasziniert. 